\title{
Chiral angle dependence of resonance window widths in $(2 n+m)$ families of single-walled carbon nanotubes
}

\author{
Ya-Ping Hsieh, ${ }^{1,2}$ Mario Hofmann, ${ }^{1}$ Hootan Farhat, ${ }^{3}$ Eduardo B. Barros, ${ }^{4}$ Martin Kalbac, ${ }^{5}$ \\ Jing Kong, ${ }^{1}$ Chi-Te Liang, ${ }^{2, a)}$ Yang-Fang Chen, ${ }^{2,6}$ and Mildred S. Dresselhaus ${ }^{1,7, a)}$ \\ ${ }^{1}$ Department of Electrical Engineering and Computer Science, Massachusetts Institute of Technology, \\ Cambridge, Massachusetts 02139-4037, USA \\ ${ }^{2}$ Department of Physics, National Taiwan University, Taipei 106, Taiwan \\ ${ }^{3}$ Department of Material Science and Engineering, Massachusetts Institute of Technology, Cambridge, \\ Massachusetts 02139-4037, USA \\ ${ }_{5}^{4}$ Departamento de Fisica, Universidade Federal do Ceara, Fortaleza, Ceara, CEP 60455-760, Brazil \\ ${ }^{5}$ J. Heyrovsky Institute of Physical Chemistry, Academy of Sciences of the Czech Republic, V.V.i., \\ Dolejskova 3, CZ-18223 Prague 8, Czech Republic \\ ${ }^{6}$ Center for Condensed Matter Sciences, National Taiwan University, Taipei 106, Taiwan \\ ${ }^{7}$ Department of Physics, Massachusetts Institute of Technology, Cambridge, Massachusetts 02139-4037, USA
}

(Received 26 January 2010; accepted 18 February 2010; published online 12 March 2010)

Raman spectra of isolated single-walled carbon nanotubes (SWNTs) were obtained for a wide range of laser excitation energies to study the resonance excitation window of the radial breathing mode feature for members of $(2 n+m)$ families. A chiral angle $(\theta)$ dependence of the resonance window width $(\Gamma)$ was observed, which is much stronger than the diameter dependence. The implications of this work on nanotube metrology are discussed. (C) 2010 American Institute of Physics.

[doi:10.1063/1.3359427]

Single wall carbon nanotubes (SWNTs) are a promising material for nanoscale electronic devices. Experimental investigation of the electron-phonon scattering mechanism that governs electron transport and its relation to the nanotube's electronic structure is a high priority in route toward developing device applications.

Raman spectroscopy has found wide application in the field of carbon nanotube characterization ${ }^{1}$ since it can provide noninvasive analysis. Resonant enhancement of the Raman signal occurs when the laser excitation energy $E_{\text {laser }}$ coincides with an electronic transition and allows spectroscopic analysis even at the single nanotube level. The dependence of the Raman intensity of each feature in the Raman spectra on $E_{\text {laser }}$ is called its resonance window or resonance profile and provides a powerful analysis tool for studying the electron relaxation in SWNTs (see Fig. 1). Furthermore, these resonance windows determine the signal from a mixture of nanotubes as generally obtained by SWNT synthesis. ${ }^{6}$ Thus a comprehensive knowledge of the resonance window for each SWNT will enable a more reliable evaluation of the (n, m) distribution that is present in a synthesized SWNT sample using a limited set of $E_{\text {laser }}$ values. ${ }^{2}$

The resonance window of each $(n, m)$ SWNT, characterized by its resonance window width, is expected to depend sensitively on its electronic structure. ${ }^{3,4}$ It will thus exhibit a dependence on both diameter $\left(d_{t}\right)$ and chiral angle $(\theta)$. We here investigate resonance windows for different SWNTs experimentally within an $(2 n+m)$ family of metallic and semiconducting M-SWNTs and S-SWNTs. We chose to study SWNTs comprising complete $(2 \mathrm{n}+\mathrm{m})$ families since they vary only weakly with $d_{t}$, while exhibiting a large variation in $\theta$ between zigzag $\left(\theta=0^{\circ}\right)$ and armchair $\left(\theta=30^{\circ}\right)$. Furthermore working with a whole $(2 n+m)$ family of SWNTs in-

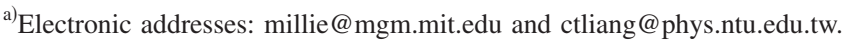

creases the accuracy of our measurements since a common trend for nanotubes of the same fundamental electronic structure can then be extracted. We here focus on the resonance window of the radial breathing mode (RBM) feature in the Raman spectrum because of its usefulness for nanotube metrology.

There has only been limited research on resonance windows of individual SWNTs. ${ }^{2,4}$ Instead, most previous Raman resonance window studies have been attempted with SWNT ensembles (either aqueous solutions ${ }^{5,6}$ or SWNT bundles ${ }^{7}$ ). Changes in the SWNT environment due to neighboring nanotubes or to surfactant wrapping introduce a broadening of the resonance window ${ }^{6}$ and thus conceal effects caused by changes of the electronic structure and are only partly applicable to individual SWNTs on a $\mathrm{SiO}_{2}$ substrate- a common device layout.

Individual SWNT samples were prepared using the electrostatic spray-assisted chemical vapor deposition method as described previously. ${ }^{8}$ Such samples are expected to contain mostly individual SWNTs. In short, SWNTs were deposited

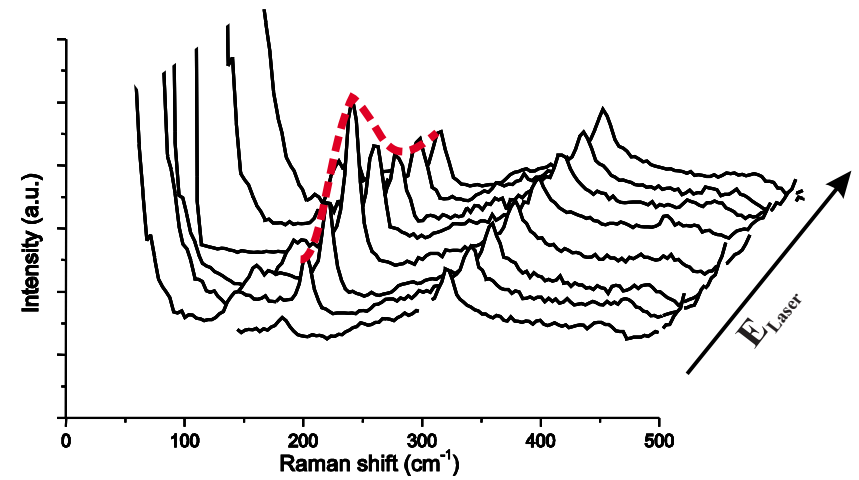

FIG. 1. (Color online) Plot of Raman spectra for various $E_{\text {laser }}$ energies. The dashed line traces the resonance window of this $(13,1)$ SWNT. 


\section{(a) Family 38}

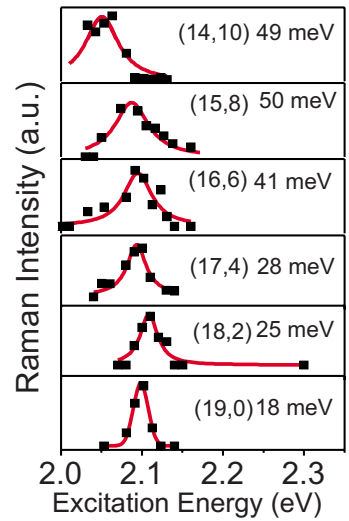

(b) Family 27
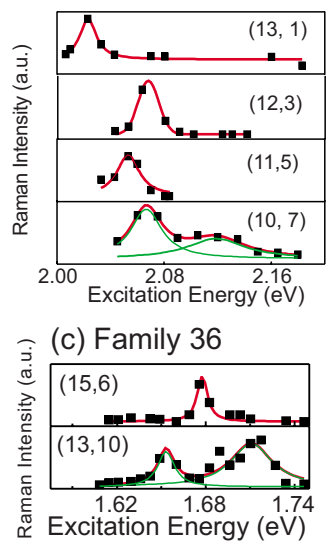

FIG. 2. (Color online) (a) Data (squares) and fitting (line) for Resonance windows for S-SWNT family 38 and for M-SWNT families 27 (b) and 36 (c), respectively. The thinner lines in (b) and (c) correspond to the upper and lower contributions of $E_{11}^{M}$ and transitions to the resonance window of metallic nanotubes (Ref. 3).

from the gas phase onto a $\mathrm{Si}$ substrate coated with a $100 \mathrm{~nm}$ $\mathrm{SiO}_{2}$ thermal oxide after the SWNTs were grown at $1000{ }^{\circ} \mathrm{C}$ using a floating ferrocene catalyst. ${ }^{8}$ From the good agreement between the $d_{t}$ distribution obtained using AFM and Raman measurements, ${ }^{8}$ we confirmed that our synthesis method produced mostly well isolated individual, nonbundled SWNTs. The low density $\left[1 \mathrm{SWNT} /\left(5 \mu \mathrm{m}^{2}\right)\right]$ of the deposited nanotubes allows measurements of the Raman resonance window of individual SWNTs to be made without the interference from the presence of other SWNTs. ${ }^{1}$

Various laser excitation lines spanning two quasicontinuous ranges from $570 \mathrm{~nm}(2.17 \mathrm{eV})$ to $610 \mathrm{~nm}(2.03$ $\mathrm{eV})$ and from $720 \mathrm{~nm}(1.72 \mathrm{eV})$ to $810 \mathrm{~nm}(1.53 \mathrm{eV})($ Dye and Ti-Sapphire Lasers, respectively) were used in this study. The experimental data for each laser excitation energy were taken by using $60 \times 80 \mu \mathrm{m}^{2}$ Raman mappings with $0.5 \mu \mathrm{m}$ resolution. Each map point contains a complete Raman spectrum. This allows us to account for errors in sample positioning during the experiment and to acquire resonance window data in parallel under identical laser conditions for several SWNTs in the same map area. A trade-off between acquisition speed and spectral resolution had to be made and laser excitation wavelengths were varied in $3 \mathrm{~nm}$ steps in data acquisition. In the investigated $E_{\text {laser }}$ ranges, the resonance windows of the $E_{11}^{M}$ and $E_{33}^{S}$ electronic transitions ${ }^{1}$ are probed in our SWNT sample.

Figure 2(a) shows the RBM resonance window for six SWNTs in the family $(2 \mathrm{n}+\mathrm{m})=38$ for many different $E_{\text {laser }}$ values. In this figure the Raman intensity is normalized to the intensity of the Si $303 \mathrm{~cm}^{-1}$ peak in each spectrum.

The plot for each SWNT exhibits a resonance window which is characterized by its peak intensity $I_{\max }$, its transition energy $\mathrm{E}\left(I_{\max }\right)$ and its resonance window width. The shape of the resonance window is determined by contributions from two peaks associated with resonances between the electronic structure and the incident or scattered photons. Since the energy separation between those contributions (the RBM phonon energy $\left.\hbar \omega_{\mathrm{RBM}}\right)$ is comparable with their peak widths $(\gamma)$, it is difficult to distinguish these two contributions to the resonance profile from each other. Experimental results furthermore suggest that there might be varying intensities of

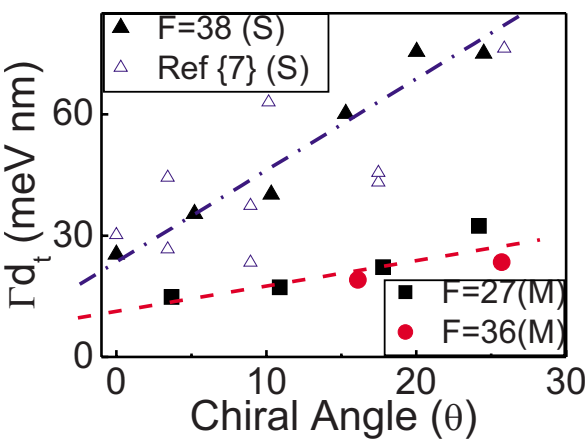

FIG. 3. (Color online) Plot of $\Gamma d_{t}$ vs $\theta$ with a linear fit to resonance window data acquired from individual M-SWNTs and S-SWNTs of this work. Data for $\Gamma d_{t}$ vs $\theta$ for the S-SWNTs from Ref. 7 is shown for comparison. (The $\Gamma$ of Ref. 7 were obtained by fitting the original data with one Lorentzian peak.)

the two peaks in the resonance window. ${ }^{9}$ This multitude of unknown parameters and the limited experimental data points make a de-convolution into two peaks of varying width and intensities not viable. We therefore here report the total full width at half maximum (FWHM) of one Lorentzian peak (capital $\Gamma$ ) and note that it not only depends on the variation of $\gamma$ and $\hbar \omega_{\mathrm{RBM}}$ but is also affected by the variation of the relative intensities of the incident and scattered photon resonance peaks.

The excitation energy $E\left(I_{\max }\right)$ at the maximum RBM intensity $I_{\max }$ together with the $\omega_{\mathrm{RBM}}$ for each SWNT gives a characteristic point on the Kataura plot of the resonant transition energy versus $\omega_{\mathrm{RBM}} \cdot{ }^{1}$ When compared with the theoretical Kataura plot calculated by Samsonidze et al. ${ }^{10}$ using the equation $\omega_{\mathrm{RBM}}=217.8 / d_{t}+15.7 \mathrm{~cm}^{-1}$ (Ref. 11), excellent agreement is found. Thus the $(\mathrm{n}, \mathrm{m})$ chirality of the investigated SWNTs and their $(2 n+m)$ family can be assigned, and both the $d_{t}$ and $\theta$ for each SWNT can be extracted.

All members of the S-SWNT family 38 have similar diameters but large changes in $\Gamma$ are observed for their respective resonance profiles in Fig. 2(a). We will subsequently correlate these variations in $\Gamma$ with changes in $\theta$.

To analyze the intrinsic $\theta$ dependence of $\Gamma$ in more detail, we have to account for the $d_{t}$ dependence of $\Gamma$. We therefore also investigated SWNTs in the $(2 n+m)$ metallic family (27 along with two members of family 36 ) whose constituents span a wider range of diameters than the investigated S-SWNT family 38 . The resulting data are shown in Figs. 2(b) and 2(c). Following theoretical modeling ${ }^{10}$ and our own experimental observations we account for changes in $\Gamma$ due to $d_{t}$ by approximating these changes with a $1 / d_{t}$ dependent term. We therefore plot in Fig. 3 the product $\Gamma d_{t}$ versus $\theta$ for the two investigated M-SWNT families, and find that the $\Gamma d_{t}$ data for both M-SWNT families with different values for $d_{t}$ and $\Gamma$ indeed collapses on to a single line with a clear $\theta$ dependence. The same is true for the S-SWNTs and in particular we see that our $\Gamma d_{t}$ data for family 38 is consistent with other experimental data obtained by Yin et $\mathrm{ll}^{7}$ for S-SWNTs from families 22 and 29. The large scatter in their data might be related to bundling effects. When extrapolating $\Gamma d_{t}$ to $\theta=0^{\circ}$ from the plot in Fig. 3, we find that the minimum profile width denoted by $\Gamma_{0} d_{t}$ for $E_{33}^{S}$ S-SWNTs $(\sim 20 \mathrm{meV} \mathrm{nm})$ is larger than that for $E_{11}^{M}$ M-SWNTs $(\sim 10 \mathrm{meV} \mathrm{nm})$. 
Theoretical modeling based on phonon-assisted electron relaxation like in Refs. 3 and 12 predicts that $\Gamma$ is larger for S-SWNTs than for M-SWNTs of the investigated transitions but it does not predict the two main findings of our experiments. First, the value of $\Gamma_{0} d_{t}$ is overestimated significantly by theory (i.e., Ref. 4 calculates a $\Gamma$ for members of Family 38 that is twice as large as our observed value). Second, the clear dependence of $\Gamma$ on $\theta$, observed experimentally in Fig. 3 , is not described by the theoretical modeling.

Our experimental findings open a new research area, and future experiments and theoretical modeling are required for explaining explain the experimental observations reported here. Future investigations have to determine if the observed dependence of $\Gamma$ on the chiral angle is an intrinsic effect not captured by theory or caused by electronic interactions of the nanotube with adsorbates or with the substrate. Studies of the influence of nanotube properties (metallicity, S-SWNT type (type I or type II), optical transition energies $E_{i i}$, and nanotube diameter $d_{t}$ ) will help to better interpret the dependence of $\Gamma d_{t}$ on $\theta$.

The observed changes of $\Gamma$ with metallicity and chiral angle have implications on applications such as the metrological analysis of carbon nanotube samples. Since most evaluations of chirality and metallicity distributions of SWNTs are performed using a limited set of laser excitation energies, only a small set of nanotubes contributes to the Raman signal. For these nanotubes, the laser energy falls within their resonance window around their transition energies. Thus the experimental evidence of a larger $\Gamma$ for S-SWNTs compared to M-SWNTs implies errors in the analysis of these M-SWNT to S-SWNT ratios since the S-SWNTs present in the sample will contribute more to the measured Raman intensity. Furthermore, the strong influence of $\theta$ on $\Gamma$ could lead to an overestimation of near armchair over near zigzag nanotubes for each metallicity. The results reported here would thus require a revision of the current methodologies used for SWNT sampling for metrological applications to be made.

In conclusion, measurements of the resonance windows for two carbon nanotube families show that the resonance window width $\Gamma$ has a clear chiral angle dependence. The findings reported in this work open a new research direction for further experimental and theoretical study of resonance windows in carbon nanotubes.

The authors thank Professors A. Jorio, R. Saito and A. Souza Filho for helpful discussions. The work is partially supported by the Materials, Structures, and Devices (MSD) Center, one of the five programs in the Focused Center Research Program of the NSC and by NSF Grant No. 0704197. Y.P.H. also acknowledges financial support from the NSC, Taiwan (Grant No. 95-2112-M-002-047-MY4), M.K. acknowledges the support from ME09060, E.B.B. acknowledges support from the NSF-CNPq (Grant No. 491083/ 2005-0) joint collaboration and CNPq Grant No. 577489/ 2008-9.

${ }^{1}$ M. S. Dresselhaus, G. Dresselhaus, R. Saito, and A. Jorio, Phys. Rep., Phys. Lett. 409, 47 (2005).

${ }^{2}$ A. Jorio, A. G. Souza, G. Dresselhaus, M. S. Dresselhaus, R. Saito, J. H. Hafner, C. M. Lieber, F. M. Matinaga, M. S. S. Dantas, and M. A. Pimenta, Phys. Rev. B 63, 245416 (2001).

${ }^{3}$ J. S. Park, Y. Oyama, R. Saito, W. Izumida, J. Jiang, K. Sato, C. Fantini, A. Jorio, G. Dresselhaus, and M. S. Dresselhaus, Phys. Rev. B 74, 165414 (2006).

${ }^{4}$ M. Steiner, M. Freitag, V. Perebeinos, J. C. Tsang, J. P. Small, M. Kinoshita, D. Yuan, J. Liu, and P. Avouris, Nat. Nanotechnol. 4, 320 (2009).

${ }^{5}$ B. C. Satishkumar, S. V. Goupalov, E. H. Haroz, and S. K. Doorn, Phys. Rev. B 74, 155409 (2006).

${ }^{6}$ C. Fantini, A. Jorio, M. Souza, M. S. Strano, M. S. Dresselhaus, and M. A. Pimenta, Phys. Rev. Lett. 93, 147406 (2004).

${ }^{7}$ Y. Yin, A. G. Walsh, A. N. Vamivakas, S. B. Cronin, A. M. Stolyarov, M. Tinkham, W. Bacsa, M. S. Unlu, B. B. Goldberg, and A. K. Swan, IEEE J. Sel. Top. Quantum Electron. 12, 1083 (2006).

${ }^{8}$ Y. P. Hsieh, M. Hofmann, H. B. Son, X. T. Jia, Y. F. Chen, C. T. Liang, M. S. Dresselhaus, and J. Kong, Nanotechnology 20, 065601 (2009).

${ }^{9}$ A. P. Shreve, E. H. Haroz, S. M. Bachilo, R. B. Weisman, S. Tretiak, S. Kilina, and S. K. Doorn, Phys. Rev. Lett. 98, 037405 (2007).

${ }^{10}$ G. G. Samsonidze, R. Saito, N. Kobayashi, A. Gruneis, J. Jiang, A. Jorio, S. G. Chou, G. Dresselhaus, and M. S. Dresselhaus, Appl. Phys. Lett. 85, 5703 (2004)

${ }^{11}$ P. T. Araujo, S. K. Doorn, S. Kilina, S. Tretiak, E. Einarsson, S. Maruyama, H. Chacham, M. A. Pimenta, and A. Jorio, Phys. Rev. Lett. 98, 067401 (2007).

${ }^{12}$ K. K. Kim, J. S. Park, S. J. Kim, H. Z. Geng, K. H. An, C. M. Yang, K. Sato, R. Saito, and Y. H. Lee, Phys. Rev. B 76, 205426 (2007). 